\title{
The Discussion of the Effects of Linear and Nonlinear Algorithms on Emotional Evaluation
}

\author{
Yang $\mathrm{Wu}^{1}$, Yu Nie ${ }^{2}$, Guang-Zhi Sun ${ }^{3}$, Zhong-Yao Yang4 \\ 1Software College of Jilin University, Changchun China \\ 2Software College of Jilin University, Changchun China \\ 3Software College of Jilin University, Changchun China \\ 4Software College of Jilin University, Changchun China

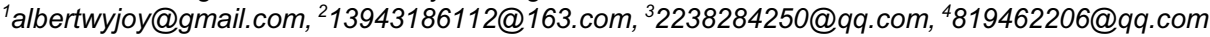

\begin{abstract}
With the continuous development of brain-computer interface technology, as a typical brainwave signal carrying brain state information, has entered the researcher's point of view. Brain waves carry detailed information about the state of the brain. [1]Therefore, the use of computers to collect and analyze EEG signals play an important role in the study of artificial intelligence. In this paper, the EEG signal of positive and negative emotions is taken as the research object.[2] Firstly, the present situation of brain wave research is introduced, and the image is experimented with the Chinese emotional image system (CAPS) of Chinese Academy of Sciences. Based on the previous work of "Emotional Evaluation Based on SVM", further data analysis was carried out using support vector machines (SVM) and linear machines with linear and nonlinear cores, and $49.2 \%$ to $62.4 \%$ accuracy were obtained respectively. This paper provides a feasible solution for the study of EEG in the field of emotional analysis.Finally, through the accuracy of the linear algorithm and the nonlinear algorithm on the data, we find that the EEG data are non-linear separable.
\end{abstract}

\section{Introduction}

Since the last century, James, Connon and other scholars found that: emotions is a change in their own body of a feeling, first physical changes, only emotional perception, any emotional generation must be accompanied by some changes in the body [3]. This is the earliest definition of emotion. Moreover, the emotional generation is determined by the hypothalamus [4]. At present, a large number of neurological and cognitive science-related research results show that emotional production and physiological activities, especially with the cortex activity is closely related to the study by the cerebral cortex activity analysis and identification of human emotional state provides a theoretical basis [6].

It is of great significance to study emotional recognition. Artificial intelligence in an indicator is to understand the human emotions, so the emotional identification of related research will promote the development of artificial intelligence. In terms of human-computer interaction, let the machine understand human emotions, will make it more accurate understanding of human intent, so as to better help humans. Emotional recognition of the application there are many, such as driver fatigue testing and cardiac care and so on.
Emotional classification, such as emotions, has not yet formed a unified theoretical understanding. Researchers through further research, found that there is still a certain correlation between emotions, such as anger and aversion sometimes appear at the same time, so there Lange two-dimensional emotional classification model [7]. The model is also the most common classification model, the use of vertical coordinates of the mood of pleasure, never like to gradually over to like; use the abscissa that the state of excitement, from the downturn to excitement.

There are two major categories of common emotion recognition methods. The first category is based on the recognition of nonphysiological signals, which include recognition of facial expressions and recognition of speech tones. The advantage is that the data acquisition is relatively simple, do not need special equipment; shortcomings cannot guarantee the reliability of emotional recognition, because it will be subjective factors. The second category is based on physiological signal recognition. At present, the physiological signals adopted at home and abroad mainly include electrooculogram (EOG), Galvanic Skin Response (GSR), Blood Volume Pulse (BVP), Electromyogram (EMG), EEG (EEG), electrocardology (Electrocardiogram, ECG) and so on.

At present, there are many studies on emotional recognition based on EEG signals [8, 9]. In the 
emotional mode of learning and classification stage, the algorithm used at home and abroad is mainly to support the vector machine. The academic community has now been able to achieve $75 \%$ of the emotional classification by some data analysis methods [10].

The main steps of emotion recognition based on EEG include: emotional induction; collection of EEG signals; pretreatment of EEG signals; feature extraction; feature dimensionality reduction; learning and classification of emotional patterns. This paper mainly through the use of machine learning in two different ways to human brain waves.

\section{Experiment}

After being processed by pretreatment algorithm such as low-pass filtering algorithm, the EEG data obtained from the psychological experiment became 8dimensional data. Before classification, we should analysis the degree of linear correlation between data in the different dimension. We used the linear correlation coefficients to quantify it. As shown in figure 1, there were different degrees of relevance between data in the different dimension. Therefore, we considered using dimension reduction algorithm. On one hand, the dimensions would be lower which made it more convenient to observe the data and because data in different dimension were not independent, we removing some features from the data through the dimension reduction algorithm would not decrease the accuracy of classification too much.

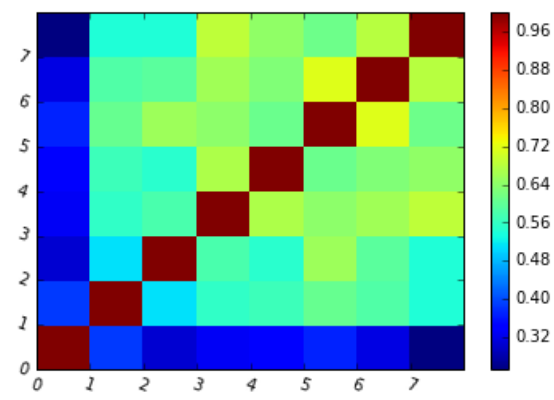

Figure 1

Before beginning our experiment, we need to observe the data distribution, so we could get a preliminary understanding. The method was that we used PCA algorithm to process the dimensional data from which we could get 3 or 2-dimensional data and we display the data in coordinates as shown in Figure 2, Figure 3 and Figure 4.

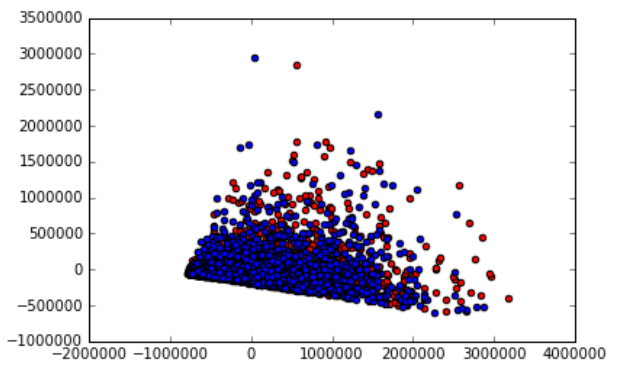

Figure 2

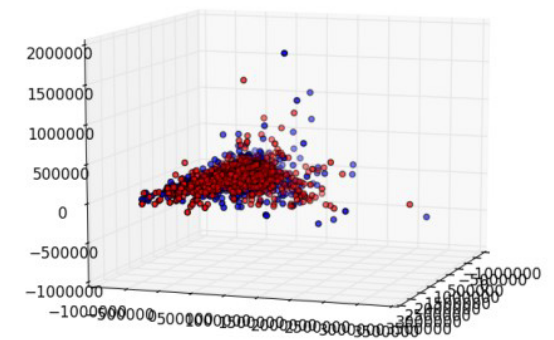

Figure 3

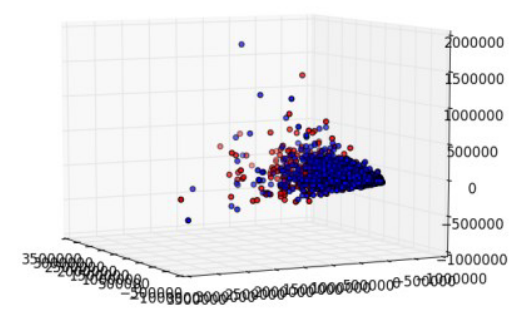

Figure 4

We found that Figure 2 could be seen as a projection from three-dimensional space to two-dimensional space of Figure 3 or Figure 4 and also know that the distribution of data was non-linear which caused the data was hard to classify. Through observed Figure 3 and Figure 4, we found the two kinds data cannot be split by a plane. Just observing more carefully, we could know the data were split by the graph like shown in Figure 5, the red point on the inside and blue point on the outside. 


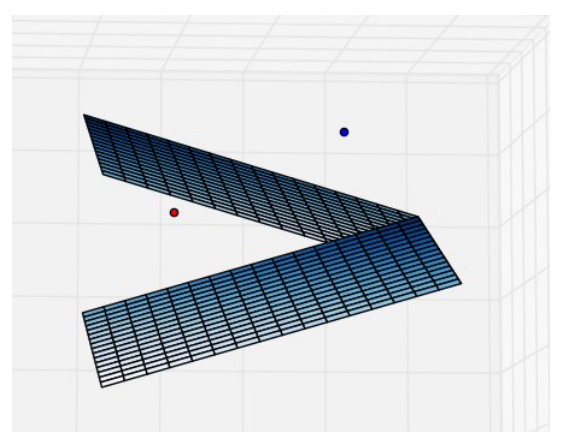

Figure 5

Based on the distributions of two-dimensional data and three-dimensional data, we estimated that the EEG data was nonlinear separable. Therefore, the classification result would not be good when we used the linear classifying algorithm such as perceptron algorithm and SVM with linear kernel algorithm. However, the classification result would be better when we used the no-linear classifying algorithm such as SVM with the no-linear kernel.

We used perceptron algorithm, SVM with linear kernel algorithm and SVM with nonlinear kernel (RBF) algorithm for experiments. We did our experiments on two sets of data. One was 8-dimensional data (the EEG data). The other was 3-dimensional data obtained through PCA algorithm. Considering the SVM algorithm, we set the parameter $\gamma$ to different values 1,10 and 20. The accuracy of classification of SVM with nonlinear kernel (RBF) algorithm on data was shown in Table 1. The accuracy of classification of SVM with linear kernel algorithm on data was shown in Table 2 . As for perceptron algorithm, we set the iterative number to different values 10,100 and 1000 . The accuracy of perceptron was shown in Table 3.

Table 1

\begin{tabular}{|l|l|l|}
\hline$\gamma$ & SVM RBF(8) & SVM RBF (3) \\
\hline 1 & 0.570 & 0.511 \\
\hline 10 & 0.624 & 0.561 \\
\hline 20 & 0.623 & 0.568 \\
\hline
\end{tabular}

Table 2

\begin{tabular}{|c|c|c|}
\hline$\gamma$ & SVM linear(8) & $\begin{array}{l}\text { SVM linear } \\
\text { (3) }\end{array}$ \\
\hline 1 & 0.506 & 0.497 \\
\hline 10 & 0.506 & 0.497 \\
\hline 20 & 0.506 & 0.497 \\
\hline
\end{tabular}

Table 3

\begin{tabular}{|l|l|l|}
\hline $\mathrm{n}$ & Perceptron(8) & $\begin{array}{l}\text { Perceptron( } \\
3)\end{array}$ \\
\hline 10 & 0.511 & 0.511 \\
\hline 100 & 0.490 & 0.506 \\
\hline 1000 & 0.492 & 0.520 \\
\hline
\end{tabular}

Though the experimental data shown in the Table1, Table 2 and Table 3, we could find on both 8dimensional data and 3-dimensional data the accuracy of classification of SVM with nonlinear kernel (RBF) algorithm is highest among the three algorithms. And SVM with linear kernel algorithm considered, its performance on 8-dimensional data was better than 3dimensional data. The accuracy of the linear algorithm was about $50 \%$. We could find the reason of it in the distribution of data. The distribution of 3-dimensional data could be seen as shown in Figure 5. The two kinds data was split by the graph. Whatever the plan decided by the algorithm was the upper plane or the lower plane or any plane, there would be many points which were classified wrongly. However, the no-linear algorithm mapped nonlinear data into high-dimensional space which made it easier to classify.

\section{Conclusion}

In this study, we obtained the EEG data and did experiments on it. Firstly, we analyzed the degree of linear correlation between data in the different dimension and we found here were different degrees of relevance between data in the different dimension. But the accuracy of SVM with nonlinear kernel (RBF) algorithm on 8-dimensional data was higher than 3dimensional data which indicated even if the data in different dimension were independent, the information in the data would lose after reducing the dimension of data. To sum up, though observing the distribution of the data and the results of experiments the accuracy of the linear algorithm was lower than that of the no-linear algorithm, we concluded that the EEG data is nonlinear separable.

\section{References}

[1] W. James. Mind 34 (1884): 188-205.

[2] W.B. Cannon. The American journal of psychology 39.1/4 (1927): 106-124.

[3] T. Dalgleish. Nature Reviews Neuroscience 5.7 (2004): 583-589.

[4] P.J. Lang, M.M. Bradleyand B.N. Cuthbert. "International affective picture system (IAPS): Technical manual and affective ratings." Gainesville, FL: The Center for Research in Psychophysiology, University of Florida (1999).

[5] H.H. Jasper. Electroencephalography and clinical neurophysiology 10 (1958): 371-375.

[6] C. Peacock. "Interfacing the standard parallel port." (1998).

[7] K. Schaaffand T. Schultz. "Towards emotion recognition from electroencephalographic signals." 2009 3rd International Conference on Affective Computing and Intelligent Interaction and Workshops. IEEE, 2009.

[8] A. Jain, K.Nandakumarand A. Ross. Pattern recognition 38.12 (2005): 2270-2285. 
[9] B. Kahn. Electroencephalogram (EEG) signal processing, wave identification, and emotion recognition. Diss. California State University, Northridge, 2015.

[10] R.M. Mehmoodand H.J. Lee. Int. J. Bio-Sci. BioTechnol. 7 (2015): 23-32. 